\title{
Health Information National Trends Survey
}

National Cancer Institute

\section{Source}

National Cancer Institute. Health Information National Trends Survey. NCI Thesaurus.

Code C20093.

A survey designed to collect nationally representative data routinely about the American public's use of cancer-related information. The survey provides updates on chang ing patterns, needs, and information opportunities in health, identifies changing communications trends and practices, assesses cancer information access and usage, provides information about how cancer risks are perceived, and offers a testbed to researchers to test new theories in health communication. 